\title{
Regenerative endodontic treatment: A systematic review of successful clinical cases
}

\author{
Faisal Alghamdi ${ }^{1}, A-F$, Mohammed Alsulaimani ${ }^{2}, B, C, E, F$ \\ ${ }^{1}$ Department of Oral Biology, Faculty of Dentistry, King Abdulaziz University, Jeddah, Saudi Arabia \\ ${ }^{2}$ Department of General Dentistry, Faculty of Dentistry, King Abdulaziz University, Jeddah, Saudi Arabia \\ A - research concept and design; $\mathrm{B}$ - collection and/or assembly of data; $\mathrm{C}$ - data analysis and interpretation; \\ $D$ - writing the article; $E$ - critical revision of the article; $F$ - final approval of the article
}

Address for correspondence

Faisal T. Alghamdi

E-mail: dr.faisal2020@hotmail.com

Funding sources

None declared

Conflict of interest

None declared

Received on September 8, 2020

Reviewed on December 16, 2020

Accepted on January 4, 2021

Published online on December 31, 2021

Cite as

Alghamdi FT, Alsulaimani MA. Regenerative endodontic treatment: A systematic review of successful clinical cases. Dent Med Probl. 2021;58(4):555-567. doi:10.17219/dmp/132181

DOI

$10.17219 / \mathrm{dmp} / 132181$

Copyright

○) 2021 by Wroclaw Medical University

This is an article distributed under the terms of the

Creative Commons Attribution 3.0 Unported License (CC BY 3.0)

(https://creativecommons.org/licenses/by/3.0/).

\begin{abstract}
This systematic review was designed to evaluate and compare successful cases of regenerative endodontic treatment (RET) in terms of etiology, diagnosis, treatment protocols, and signs of success. An electronic search was performed in the PubMed and Google Scholar databases. The search was completed by 2 independent reviewers following the Preferred Reporting Items for Systematic reviews and Meta-Analyses (PRISMA) guidelines. All in vivo studies in humans that reported at least 1 successful case of RET were included in this review. Successful RET cases were defined as any case that involved the absence of clinical signs/symptoms of periapical inflammation and the presence of continued root maturation, especially apical closure, after the completion of the initial RET. A total of 250 successful cases of RET from 18 studies were selected in this review. A total of 98 (39\%) successful cases were detected at least 2.5 years after the initiation of RET. A total of 239 (96\%) successful RET cases were presented with the healing/absence of periapical lesions, and no further treatment was required. Furthermore, $45 \%$ of the successful RET cases showed root development maturation (stage V). Finally, the clinical outcomes of these RET cases are presented in this systematic review. Prudent case selection and excellent operative protocols are considered to be essential to achieve successful RET outcomes. Future studies are needed to identify a variety of relevant data, including preoperative, intraoperative and postoperative factors, in order to provide a better understanding of successful cases after RET.
\end{abstract}

Keywords: endodontic regeneration, immature necrotic teeth, outcome assessment, systematic review 


\section{Introduction}

Regenerative endodontics has been suggested as the appropriate treatment approach for immature, necrotic-pulp permanent teeth cases. Most clinical regenerative endodontic treatment (RET) cases reported in the literature have presented promising clinical results. ${ }^{1,2}$ Radiographic evidence of periapical healing and the lack of clinical signs and symptoms have been recognized as the primary indicators of successful RET. ${ }^{3}$ Also, increased root wall thickness and/or length of the immature root, similarly as recovering the vitality of the tooth, have been recognized as additional goals of RET and are associated with a high rate of success. $^{3}$ Current endodontic regeneration is frequently referred to as "revascularization". This means cleaning the root canal with the use of an antibiotic mixture and irritating the root apex tissue to create a blood clot inside the root canal that works as a natural scaffold, and to assist pulp-dentin stem cell proliferation and differentiation. ${ }^{4-6}$

Conventional endodontic therapy includes the disinfection, debridement, and subsequent obturation of the root canal system with biologically based materials to replace the function of the organ and the live tissue within the diseased system as an important objective of regeneration in the body. ${ }^{4,7,8}$ Regenerative endodontics or "regeneration" (the previous terminology also included "revascularization") consists in performing biologically based procedures to replace the necrotic tissue and create a new tooth structure. ${ }^{4}$ Additional benefits of achieving this goal are continued root development and/or apical closure (root end development), and ultimately, avoiding "traditional" root canal therapy. For regeneration, the available cells of the body are invigorated to regrow the missing tissue. Stem cells, tissue scaffolds, growth factors, and other ingredients that can be introduced into the root canal system are beneficial adjuncts for the regeneration of the pulp and further development of the roots. ${ }^{4,8-11}$

After the redefinition of RET in its new concept, evidence-based clinical outcomes have been extracted from case reports/series with favorable outcomes. However, the main apprehension related to this level of evidence is that it may not factually represent the true results of RET, considering that unfavorable outcomes are underreported in most cases. In the last 5 years, numerous prospective and retrospective clinical studies linked to RET have been published in the literature. ${ }^{1,2,12,13}$ These types of studies contribute to a higher level of evidence related to successful RET outcomes and present a relatively more accurate depiction of successful RET cases. One review study from 2018 concluded that a successful regenerative procedure was achieved by the regeneration of the pulp with root end closure as well as the healing/absence of periapical pathology, and no further treatment being required. ${ }^{14}$ However, if signs or symptoms of disease or necrosis of the new pulp/pulp-like tissue occur, any additional apical closure from the regenerative endodontic procedure can promote a more predictable outcome, should root canal therapy later be required. Thus, even "unsuccessful" regeneration can still be beneficial, as any progress from the initial immature-apex state must be acknowledged as a victory. ${ }^{2,14}$

Previous clinical studies and case reports have revealed positive clinical results of the regenerative therapy of immature necrotic permanent teeth. ${ }^{5,15-22}$ Managing RET cases requires good treatment planning, and awareness to determine multiple challenges and to prevent any complications that may occur. The presence of an already compromised tooth with necrotic pulp and an open apex represents a major challenge. Other challenges regard the signs and symptoms of healing periapical lesions which occurred after a successful attempt at RET, such as root maturation and/or the resolution of apical infection. To date, a few available review studies from among the peer-reviewed literature have focused on the success rates of different RET cases without covering most of the different factors influencing RET outcomes associated with successful cases. ${ }^{23-25}$ Therefore, the present systematic review aimed to systematically collect, compare and evaluate all successful cases of RET mentioned in the literature. It involved highlighting the etiology for the initiation of RET in these cases, the initial diagnosis of successful cases, the treatment protocols used in these cases, the signs of success, and the time needed to successfully treat immature necrotic teeth.

\section{Material and methods}

This study was conducted by 2 independent reviewers following the Preferred Reporting Items for Systematic reviews and Meta-analyses (PRISMA) guidelines. ${ }^{26}$

\section{Research question}

According to the PRISMA guidelines, the following research question was framed:

"Can successful clinical regenerative endodontic treatment cases be used as strong evidence and a promising future approach in the field of endodontic treatment to treat patients with immature necrotic pulp teeth?"

\section{Information sources}

An electronic search for articles published in the English language in the years 2015-2020 was performed using the PubMed and Google Scholar databases. 


\section{Literature search strategy}

The literature search strategy was carried out in July 2020. The search was performed following the PRISMA guidelines, using 2 electronic databases - PubMed and Google Scholar. The electronic search used the Medical Subject Headings $(\mathrm{MeSH})$ terms, which were combined with the Boolean operators ("AND" and "OR"). The following keywords were used: 'immature teeth' OR 'immature tooth' OR 'immature dentition' AND 'pulp revascularization' OR 'pulpal regeneration' OR 'pulp revitalization' OR 'root canal revascularization' OR 'root maturation' OR 'regenerative endodontic' OR 'regenerative endodontic therapy' OR 'regenerative endodontic treatment' OR 'regenerative endodontic procedure' AND 'blood clot' OR 'platelet-rich fibrin' OR 'platelet-rich plasma' AND 'calcified barrier' OR 'apical closure' OR 'root end formation' OR 'root apex closure.' The detailed description of the search strategy is outlined in Table 1.

This systematic review used the available full-text articles that illustrated the relevant searching of PubMed and Google Scholar. Other databases, such as Scopus, CINAHL (EBSCOhost), Web of Science, and ProQuest Dissertations \& Theses (PQDT), were not included in the search strategy, since both the PubMed and Google Scholar databases expand beyond International Scientific Indexing (ISI)-listed journals to include additional scholarly sources, such as non-ISI journals and non-indexed articles that may not be shown in specific databases like Scopus, CINAHL (EBSCOhost) or Web of Science.

The following inclusion criteria were applied to determine which articles would be accepted for the study:

- all in vivo studies in humans in which RET was performed;

- studies published in the English language;

- studies published between 2015 and 2020;

- studies that reported at least 1 successful case of RET.

Due to the lack of consensus in the literature as to defining successful outcomes of RET, the success of RET was defined in the present systematic review as any case of RET that included significant root development maturation as well as the healing/absence of periapical pathology, and no further treatment being required.
Articles that met any of the following criteria were excluded:

- review articles;

- in vitro studies;

- editorial, thesis and personal opinion articles;

- articles that did not report any successful RET cases;

- articles that illustrated clinical relevance about RET by means of percentages and samples taken from non-human sources.

\section{Critical appraisal}

Both reviewers independently screened the titles and abstracts of the retrieved articles according to the eligibility criteria as well as the PRISMA guidelines. Any disagreement was resolved through discussion among the 2 reviewers until consensus was reached.

\section{Data extraction}

The studies were initially selected by reading their respective titles and abstracts. Subsequently, the full texts of the selected articles were analyzed and organized into standardized Microsoft Office Excel worksheets by both reviewers on an independent basis. The following variables were considered: title; abstract; material and methods; type of publication; and main results.

\section{Data items}

Data items from the studies was collected and organized into a table with the following information: author and year, study design, number of successful cases, age and gender of the patients with regard to successful cases, tooth type, etiology, diagnosis, treatment protocol used (i.e., irrigation, intracanal medicaments and the inclusion of any specific scaffold), number of visits needed to finish the treatment, time elapsed between finishing RET and the reported success, signs of success, and root development stage (Cvek's classification).

Table 1. Search strategy used in the present study

\begin{tabular}{|c|c|c|}
\hline $\begin{array}{c}\text { Database, } \\
\text { search characteristics }\end{array}$ & Search strategy & Results \\
\hline $\begin{array}{l}\text { PubMed } \\
\text { - from inception } \\
\text { up to July } 31,2020 \\
\text { - all fields } \\
\text { - with no limits }\end{array}$ & $\begin{array}{l}\text { \#1 S'immature teeth'OR 'immature tooth' OR 'immature dentition' } \\
\text { \#2 'pulp revascularization' OR 'pulpal regeneration' OR 'pulp revitalization' OR 'root canal revascularization' OR 'root } \\
\text { maturation' OR 'regenerative endodontic'OR 'regenerative endodontic therapy' OR 'regenerative endodontic } \\
\text { treatment'OR 'regenerative endodontic procedure' } \\
\text { \#3 'blood clot'OR 'platelet-rich fibrin' OR 'platelet-rich plasma' } \\
\text { \#4 'calcified barrier' OR 'apical closure' OR 'root end formation' OR 'root apex closure' } \\
\text { \#5 \#1 AND \#2 AND \#3 AND \#4 }\end{array}$ & $\# 5=267$ \\
\hline $\begin{array}{l}\text { Google Scholar } \\
\text { - from inception } \\
\text { up to July } 31,2020 \\
\text { - all text - TX } \\
\text { - with no limits }\end{array}$ & $\begin{array}{c}\text { 'immature teeth' OR 'immature tooth' OR 'immature dentition' AND 'pulp revascularization' OR 'pulpal regeneration' OR } \\
\text { 'pulp revitalization' OR 'root canal revascularization' OR 'root maturation' OR 'regenerative endodontic' OR 'regenerative } \\
\text { endodontic therapy' OR 'regenerative endodontic treatment' OR 'regenerative endodontic procedure' AND 'blood clot' } \\
\text { OR 'platelet-rich fibrin' OR 'platelet-rich plasma' AND 'calcified barrier' OR 'apical closure' OR 'root end formation' OR 'root } \\
\text { apex closure' }\end{array}$ & 2,090 \\
\hline
\end{tabular}




\section{Assessment of the risk of bias of the included studies}

The assessment of the risk of bias was performed using the criteria suggested in the Cochrane Handbook for Systematic Reviews of Interventions (Version 5.1.0). ${ }^{27}$ The Cochrane Collaboration recommends a specific tool for assessing the risk of bias in each included study. The 2 review authors determined the risk of bias of the included studies during the process of data extraction. The risk of bias assessment tool includes 7 specific domains: random sequence generation; allocation concealment; blinding of participants and personnel; blinding of outcome assessment; incomplete outcome data; selective reporting; and other bias.

Each domain was assessed as 'low risk', 'unclear risk' or 'high risk'. The overall risk of bias associated with each study was evaluated as follows:

- low risk of bias: all domains were assessed as 'low risk';

- unclear risk of bias: at least 1 domain was assessed as 'unclear risk';

- high risk of bias: at least 1 domain was assessed as 'high risk'.

\section{Synthesis of the results}

As mentioned above, tables were prepared with the relevant data included as data items.

The following types of outcomes were measured:

- primary outcomes: elimination of clinical symptoms (pain, swelling and the sinus tract); reduction in radiographic evidence of the presence of apical pathology;

- secondary outcomes: root development, defined as an increase in root length and root wall thickness.

\section{Statistical analysis}

Due to the heterogeneity between the included studies, no meta-analysis could be conducted. Therefore, only parametric data involving the age of the patients for all the included studies is provided as mean and standard deviation $(M \pm S D)$. The descriptive evaluation of the findings is also shown.

\section{Results}

\section{Study selection}

A total of 2,357 studies were initially obtained through the keywords, using the databases. Of those, 1,894 were deleted after reading the title and the abstract, as they displayed either duplicity or an unrelated topic. After applying the inclusion and exclusion criteria, 185 articles were assessed for eligibility. Finally, 18 papers were selected to be included in this review. The flow chart of this systematic review is illustrated in Fig. 1.

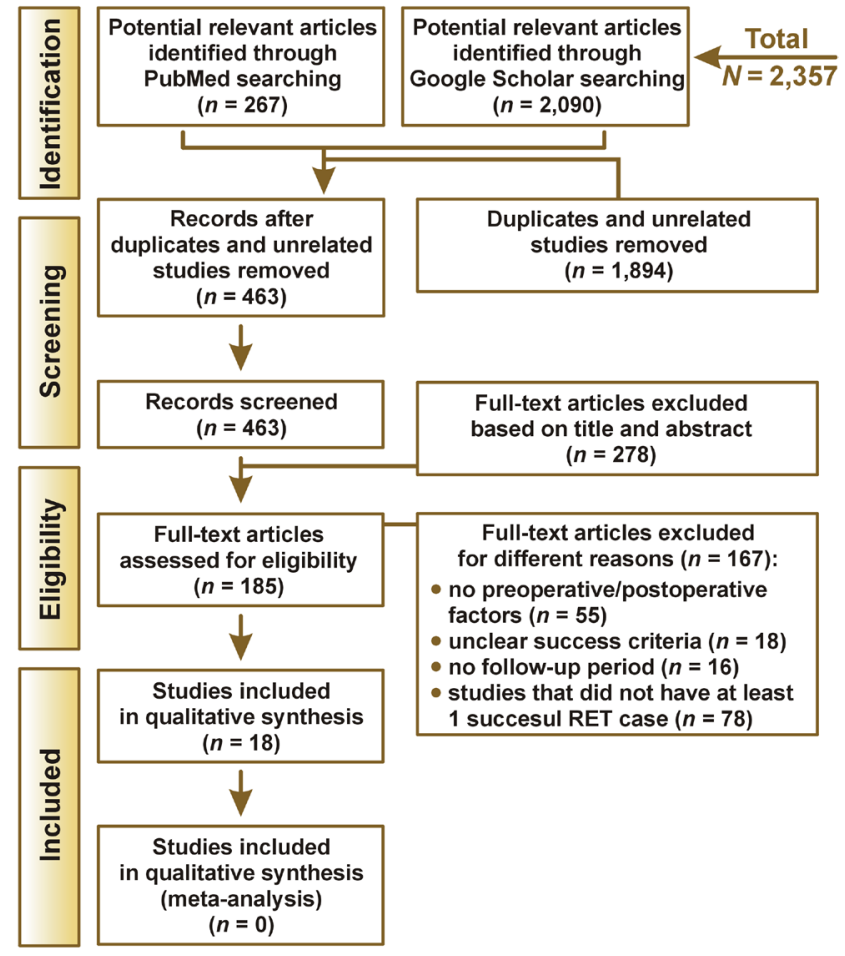

Fig. 1. Flow chart of the search strategy used in this systematic review

\section{Study characteristics}

The search ultimately included 18 human studies that satisfied both the inclusion and exclusion criteria and had been conducted within the last 6 years. These studies compared the different factors influencing RET outcomes for successful cases. The 18 included studies had a total sample of 250 successful cases of RET. ${ }^{13,28-44}$ The types of studies included in this systematic review were as follows: 7 case reports/case series ${ }^{28,30,35-37,41,44}$; 3 prospective studies ${ }^{33,39,43}$; 5 retrospective studies ${ }^{31,32,34,38,40}$; and 3 randomized clinical trials (RCTs). ${ }^{13,29,42}$ The number of successful RET cases ranged from 1 to 45 , with the total number of 250 . The distribution of these 250 cases was as follows: 10 were extracted from case reports/case series; 74 were identified from prospective studies; 112 were identified from retrospective studies; and 54 cases were identified from RCTs (Table 2). The informative description of all included studies and the different factors influencing RET outcomes for successful cases are summarized in Table 2 and Table 3.

\section{Primary outcomes}

Preoperatively, all of the 250 successful cases of RET included across the 18 studies illustrated pretreatment radiographic evidence of periapical pathology. ${ }^{13,28-44}$ Postoperatively, clinical signs indicative of infection (pain, swelling and the sinus tract) were absent from 239 (96\%) of the RET cases. There was a trend toward a reduction in the size of periapical pathology early after the initiation of RET (reported at less than 1 year) and the complete absence of periapical pathology after at least 2.5 years (Table 3 ). 
Table 2. Summary of the preoperative factors of success and the etiology of regenerative endodontic treatment (RET) cases included in the present systematic review

\begin{tabular}{|c|c|c|c|c|c|c|c|c|}
\hline Authors & Year & Study design & $\begin{array}{c}\text { Number } \\
\text { of successful } \\
\text { cases } \\
\text { in the study }\end{array}$ & $\begin{array}{l}\text { Gender } \\
(n)\end{array}$ & $\begin{array}{c}\text { Age of patients } \\
\text { [years] } \\
\text { (for } n \neq 1 \\
M \pm S D \\
\text { or range) }\end{array}$ & Tooth type & Etiology & Diagnosis \\
\hline Alasqah et al. ${ }^{28}$ & 2020 & case report & 1 & male & 8 & $\begin{array}{l}\text { posterior tooth } \\
\text { (mandibular } \\
\text { first molar) }\end{array}$ & dental caries & $\begin{array}{l}\text { necrotic pulp } \\
\text { with asymptomatic } \\
\text { apical periodontitis. }\end{array}$ \\
\hline Rizk et al. ${ }^{29}$ & 2019 & $\mathrm{RCT}$ & 26 & $\begin{array}{c}\text { male } \\
(14) \\
\text { female } \\
(12)\end{array}$ & $9.08 \pm 1.04$ & $\begin{array}{c}\text { anterior teeth } \\
\text { (maxillary } \\
\text { permanent incisors) }\end{array}$ & $\begin{array}{c}\text { trauma } \\
\text { (13) } \\
\text { dental caries } \\
\text { (13) }\end{array}$ & $\begin{array}{l}\text { necrotic pulp } \\
\text { with or without } \\
\text { apical pathosis }\end{array}$ \\
\hline Ajram et al. ${ }^{30}$ & 2019 & case report & 1 & female & 7 & $\begin{array}{l}\text { posterior tooth } \\
\text { (mandibular } \\
\text { first molar) }\end{array}$ & dental caries & $\begin{array}{c}\text { necrotic pulp } \\
\text { with symptomatic } \\
\text { apical periodontitis }\end{array}$ \\
\hline Botero et al. ${ }^{13}$ & 2017 & $\mathrm{RCT}$ & 9 & $\begin{array}{c}\text { male } \\
(5) \\
\text { female } \\
(4)\end{array}$ & $9.50 \pm 2.73$ & $\begin{array}{l}\text { anterior teeth } \\
\text { (8) } \\
\text { posterior toot } \\
\text { (1) }\end{array}$ & $\begin{array}{c}\text { trauma } \\
\text { (8) } \\
\text { dental caries } \\
(1)\end{array}$ & necrotic pulp \\
\hline $\begin{array}{l}\text { Linsuwanont } \\
\text { et al. }{ }^{31}\end{array}$ & 2017 & $\begin{array}{l}\text { retrospective } \\
\text { study }\end{array}$ & 13 & NR & $12.00 \pm 1.41$ & $\begin{array}{l}\text { anterior teeth } \\
\text { (4) } \\
\text { posterior teeth } \\
\text { (9) }\end{array}$ & $\begin{array}{c}\text { trauma } \\
(5) \\
\text { dental caries } \\
(2) \\
\text { dens } \\
\text { evaginatus } \\
\text { (6) }\end{array}$ & $\begin{array}{c}\text { necrotic pulp with } \\
\text { an acute apical abscess } \\
\text { (1) } \\
\text { necrotic pulp with } \\
\text { a chronic apical abscess } \\
\text { (5) } \\
\text { necrotic pulp } \\
\text { with asymptomatic } \\
\text { apical periodontitis } \\
(6) \\
\text { necrotic pulp } \\
\text { with symptomatic } \\
\text { apical periodontitis } \\
\text { (1) }\end{array}$ \\
\hline Peng et al. ${ }^{32}$ & 2017 & $\begin{array}{l}\text { retrospective } \\
\text { study }\end{array}$ & 45 & NR & $10.8 \pm 1.92$ & NA & NR & $\begin{array}{c}\text { necrotic pulp } \\
\text { with apical pathosis }\end{array}$ \\
\hline Chan et al. ${ }^{33}$ & 2017 & $\begin{array}{l}\text { prospective } \\
\text { study }\end{array}$ & 26 & NR & $9.20 \pm 2.41$ & NA & NR & $\begin{array}{l}\text { necrotic pulp } \\
\text { with or without } \\
\text { apical pathosis }\end{array}$ \\
\hline $\begin{array}{l}\text { Silujjai and } \\
\text { Linsuwanont }^{34}\end{array}$ & 2017 & $\begin{array}{l}\text { retrospective } \\
\text { study }\end{array}$ & 13 & NR & $16.10 \pm 10.32$ & $\begin{array}{c}(4) \\
\text { anterior teeth } \\
(4) \\
\text { posterior teeth } \\
(9-7 \text { premolars and } \\
2 \text { molars })\end{array}$ & $\begin{array}{c} \\
\text { trauma } \\
\text { (4) } \\
\text { dental caries } \\
\text { (2) } \\
\text { dens } \\
\text { evaginatus } \\
\text { (7) }\end{array}$ & $\begin{array}{c}\text { necrotic pulp with } \\
\text { an acute apical abscess } \\
(1) \\
\text { necrotic pulp with } \\
\text { a chronic apical abscess } \\
(6) \\
\text { necrotic pulp } \\
\text { with asymptomatic } \\
\text { apical periodontitis } \\
\text { (5) } \\
\text { necrotic pulp } \\
\text { with symptomatic } \\
\text { apical periodontitis } \\
\text { (1) }\end{array}$ \\
\hline Moodley et al. ${ }^{35}$ & 2017 & case report & 1 & male & 10 & $\begin{array}{l}\text { anterior tooth } \\
\text { (maxillary } \\
\text { central incisor) }\end{array}$ & dental fracture & $\begin{array}{c}\text { necrotic pulp with } \\
\text { an acute apical abscess }\end{array}$ \\
\hline $\begin{array}{l}\text { Timmerman } \\
\text { and Parashos }{ }^{36}\end{array}$ & 2017 & case report & 1 & male & 16 & $\begin{array}{l}\text { anterior tooth } \\
\text { (maxillary } \\
\text { lateral incisor) }\end{array}$ & $\begin{array}{c}\text { dens } \\
\text { invaginatus }\end{array}$ & $\begin{array}{l}\text { previously initiated } \\
\text { therapy with } \\
\text { asymptomatic apical } \\
\text { periodontitis }\end{array}$ \\
\hline $\begin{array}{l}\text { Topçuoğlu and } \\
\text { Topçuoğlu }{ }^{37}\end{array}$ & 2016 & case report & 3 & $\begin{array}{c}\text { male } \\
(1) \\
\text { female } \\
(2)\end{array}$ & $8.30 \pm 0.57$ & $\begin{array}{l}\text { posterior teeth } \\
\text { (mandibular } \\
\text { first molars) }\end{array}$ & NR & $\begin{array}{c}\text { necrotic pulp } \\
\text { with apical pathosis }\end{array}$ \\
\hline $\begin{array}{l}\text { Chen and } \\
\text { Chen }^{38}\end{array}$ & 2016 & $\begin{array}{l}\text { retrospective } \\
\text { study }\end{array}$ & 16 & NR & $10.90 \pm 0.98$ & $\begin{array}{l}\text { posterior teeth } \\
\text { (premolars) }\end{array}$ & $\begin{array}{c}\text { dens } \\
\text { evaginatus }\end{array}$ & $\begin{array}{c}\text { necrotic pulp } \\
\text { with apical pathosis }\end{array}$ \\
\hline
\end{tabular}




\begin{tabular}{|c|c|c|c|c|c|c|c|c|}
\hline Authors & Year & Study design & $\begin{array}{l}\text { Number } \\
\text { of successful } \\
\text { cases } \\
\text { in the study }\end{array}$ & $\begin{array}{l}\text { Gender } \\
\text { (n) }\end{array}$ & $\begin{array}{c}\text { Age of patients } \\
\text { [years] } \\
\text { (for } n \neq 1 \\
M \pm S D \\
\text { or range) }\end{array}$ & Tooth type & Etiology & Diagnosis \\
\hline Estefan et al. ${ }^{39}$ & 2016 & $\begin{array}{l}\text { prospective } \\
\text { study }\end{array}$ & 33 & $\begin{array}{l}\text { male } \\
(19) \\
\text { female } \\
(14)\end{array}$ & $13.20 \pm 3.06$ & $\begin{array}{l}\text { anterior teeth } \\
\text { (maxillary } \\
\text { incisors) }\end{array}$ & NR & $\begin{array}{l}\text { necrotic pulp } \\
\text { with or without } \\
\text { apical pathosis }\end{array}$ \\
\hline Bukhari et al. ${ }^{40}$ & 2016 & $\begin{array}{l}\text { retrospective } \\
\text { study }\end{array}$ & 25 & NR & $8-31$ & $\begin{array}{c}\text { anterior teeth } \\
(19) \\
\text { posterior teeth } \\
(6-5 \text { premolars and } \\
1 \text { molar })\end{array}$ & $\begin{array}{c}\text { trauma } \\
\text { (18) } \\
\text { dental caries } \\
\text { (4) } \\
\text { dens } \\
\text { invaginatus } \\
\text { (3) }\end{array}$ & $\begin{array}{l}\text { necrotic pulp } \\
\text { with or without } \\
\text { apical pathosis }\end{array}$ \\
\hline Nosrat et al. ${ }^{41}$ & 2015 & case report & 2 & $\begin{array}{l}\text { male } \\
(1) \\
\text { female } \\
(1)\end{array}$ & $9.50 \pm 0.70$ & $\begin{array}{l}\text { posterior teeth } \\
\text { (maxillary } \\
\text { first premolars) }\end{array}$ & NR & $\begin{array}{c}\text { necrotic pulp } \\
\text { without apical pathosis }\end{array}$ \\
\hline Bezgin et al. ${ }^{42}$ & 2015 & RCT & 19 & $\begin{array}{l}\text { male } \\
(10) \\
\text { female } \\
(9)\end{array}$ & $10.1 \pm 1.85$ & $\begin{array}{c}\text { anterior teeth } \\
\text { (13 maxillary incisor } \\
\text { teeth) } \\
\text { posterior teeth } \\
\text { (6-1 maxillary } \\
\text { second premolar } \\
\text { and } 5 \text { mandibular } \\
\text { premolars) }\end{array}$ & $\begin{array}{l}\text { trauma } \\
\text { (13) } \\
\text { dental caries } \\
\text { (6) }\end{array}$ & $\begin{array}{l}\text { necrotic pulp } \\
\text { with or without } \\
\text { apical pathosis }\end{array}$ \\
\hline Narang et al. ${ }^{43}$ & 2015 & $\begin{array}{l}\text { prospective } \\
\text { study }\end{array}$ & 15 & NR & below 20 & NA & NR & $\begin{array}{c}\text { necrotic pulp } \\
\text { with or without apical } \\
\text { pathosis }\end{array}$ \\
\hline McCabe $^{44}$ & 2015 & case report & 1 & female & 7 & $\begin{array}{l}\text { anterior tooth } \\
\text { (maxillary } \\
\text { central incisor) }\end{array}$ & trauma & $\begin{array}{c}\text { necrotic pulp } \\
\text { with symptomatic } \\
\text { apical periodontitis }\end{array}$ \\
\hline
\end{tabular}

RCT - randomized clinical trial; NR - not reported; NA - not applicable.

Table 3. Summary of the intraoperative and postoperative factors of success of regenerative endodontic treatment (RET) cases included in the present systematic review

\begin{tabular}{|c|c|c|c|c|c|c|c|c|}
\hline Study & Signs of success & $\begin{array}{c}\text { Root } \\
\text { development } \\
\text { stage } \\
\text { (Cvek's } \\
\text { classification) }\end{array}$ & $\begin{array}{l}\text { Irrigation } \\
\text { type }\end{array}$ & $\begin{array}{c}\text { Type of } \\
\text { medicament }\end{array}$ & $\begin{array}{l}\text { Number } \\
\text { of visits }\end{array}$ & $\begin{array}{c}\text { Capping } \\
\text { material } \\
\text { used }\end{array}$ & $\begin{array}{l}\text { Scaffold } \\
\text { used }\end{array}$ & $\begin{array}{c}\text { Time } \\
\text { between } \\
\text { the initiation } \\
\text { of RET } \\
\text { and success }\end{array}$ \\
\hline Alasqah et al. ${ }^{28}$ & $\begin{array}{l}\text { periapical healing and complete } \\
\text { root formation }\end{array}$ & stage $V$ & $\begin{array}{c}1.5 \% \mathrm{NaOCl} \\
\text { and } \\
17 \% \text { EDTA }\end{array}$ & $\begin{array}{l}\text { antibiotic } \\
\text { and } \mathrm{Ca}(\mathrm{OH})_{2}\end{array}$ & multiple & MTA & blood clot & 2 years \\
\hline Rizk et al. ${ }^{29}$ & $\begin{array}{l}\text { completed development of the } \\
\text { root apex }\end{array}$ & stage $V$ & $2 \% \mathrm{NaOCl}$ & antibiotic & multiple & MTA & $\begin{array}{l}\text { blood clot } \\
\text { (13) } \\
\text { PRP } \\
\text { (13) }\end{array}$ & 1 year \\
\hline Ajram et al. ${ }^{30}$ & $\begin{array}{c}\text { complete periapical healing and } \\
\text { apical closure }\end{array}$ & stage V & $\begin{array}{c}10 \mathrm{~mL} \\
\text { sterile saline } \\
\text { and } \\
\text { 20\% EDTA }\end{array}$ & $\mathrm{Ca}(\mathrm{OH})_{2}$ & multiple & MTA & blood clot & 2 years \\
\hline Botero et al. ${ }^{13}$ & apical closure & stage V & $\begin{array}{c}2.5 \% \mathrm{NaOCl} \\
\text { and } \\
17 \% \text { EDTA }\end{array}$ & $\mathrm{Ca}(\mathrm{OH})_{2}$ & single & MTA & blood clot & 1 year \\
\hline
\end{tabular}




\begin{tabular}{|c|c|c|c|c|c|c|c|c|}
\hline Study & Signs of success & $\begin{array}{c}\text { Root } \\
\text { development } \\
\text { stage } \\
\text { (Cvek's } \\
\text { classification) }\end{array}$ & $\begin{array}{l}\text { Irrigation } \\
\text { type }\end{array}$ & $\begin{array}{c}\text { Type of } \\
\text { medicament }\end{array}$ & $\begin{array}{l}\text { Number } \\
\text { of visits }\end{array}$ & $\begin{array}{l}\text { Capping } \\
\text { material } \\
\text { used }\end{array}$ & $\begin{array}{l}\text { Scaffold } \\
\text { used }\end{array}$ & $\begin{array}{l}\text { Time } \\
\text { between } \\
\text { the initiation } \\
\text { of RET } \\
\text { and success }\end{array}$ \\
\hline Linsuwanont et al. ${ }^{31}$ & $\begin{array}{l}\text { continued root development with } \\
\text { apical closure in most cases }\end{array}$ & $\begin{array}{l}\text { stage IV } \\
(6) \\
\text { stage V } \\
(7)\end{array}$ & $\begin{array}{c}\mathrm{NaOCl} \\
\text { and EDTA }\end{array}$ & $\begin{array}{l}\text { antibiotic or } \\
\mathrm{Ca}(\mathrm{OH})_{2}\end{array}$ & multiple & MTA & blood clot & $1-8$ years \\
\hline Peng et al. ${ }^{32}$ & $\begin{array}{l}\text { resolution of periapical lesions and } \\
\text { various levels of root development } \\
\text { with apical closure or near closure }\end{array}$ & NA & $\begin{array}{l}5.25 \% \\
\mathrm{NaOCl}\end{array}$ & antibiotic & multiple & $\begin{array}{l}\text { GIC } \\
(24) \\
\text { MTA } \\
(21)\end{array}$ & blood clot & $2.5-3$ years \\
\hline Chan et al. ${ }^{33}$ & $\begin{array}{l}\text { resolution of periapical lesions and } \\
\text { various levels of root development } \\
\text { with apical closure or near closure } \\
\text { in most cases }\end{array}$ & $\begin{array}{l}\text { stage III } \\
\quad(4) \\
\text { stage IV } \\
(14) \\
\text { stage V } \\
(8)\end{array}$ & $\begin{array}{l}5.25 \% \\
\mathrm{NaOCl}\end{array}$ & antibiotic & multiple & MTA & blood clot & 2.5 years \\
\hline $\begin{array}{l}\text { Silujjai and } \\
\text { Linsuwanont }^{34}\end{array}$ & $\begin{array}{l}\text { resolution of periapical lesions and } \\
\text { various levels of root development }\end{array}$ & $\begin{array}{l}\text { stage III } \\
\quad(4) \\
\text { stage IV } \\
\quad(4) \\
\text { stage V } \\
(5)\end{array}$ & $\begin{array}{l}1.5-2.5 \% \\
\mathrm{NaOCl} \\
\text { and } \\
17 \% \text { EDTA }\end{array}$ & $\begin{array}{l}\text { antibiotic or } \\
\mathrm{Ca}(\mathrm{OH})_{2}\end{array}$ & multiple & MTA & blood clot & 3.5 years \\
\hline Moodley et al. ${ }^{35}$ & $\begin{array}{l}\text { resolution of apical radiolucency } \\
\text { with complete apical closure }\end{array}$ & stage V & $\begin{array}{l}1.5 \% \mathrm{NaOCl} \\
\text { and } \\
17 \% \text { EDTA }\end{array}$ & $\begin{array}{c}\text { antibiotic } \\
\text { and } \mathrm{Ca}(\mathrm{OH})_{2}\end{array}$ & multiple & MTA & blood clot & $2-5$ months \\
\hline $\begin{array}{l}\text { Timmerman and } \\
\text { Parashos }\end{array}$ & $\begin{array}{l}\text { complete periapical healing and } \\
\text { complete apex formation }\end{array}$ & stage V & $\begin{array}{l}1 \% \mathrm{NaOCl} \\
\text { and } \\
15 \% \text { EDTA }\end{array}$ & $\mathrm{Ca}(\mathrm{OH})_{2}$ & multiple & MTA & blood clot & 3 years \\
\hline $\begin{array}{l}\text { Topçuoğlu and } \\
\text { Topçuoğlu } 37\end{array}$ & $\begin{array}{c}\text { absence of periapical lesions and } \\
\text { evidence of the apical closure of all } \\
\text { teeth }\end{array}$ & stage V & $\begin{array}{l}2.5 \% \mathrm{NaOCl} \\
\text { and } \\
17 \% \text { EDTA }\end{array}$ & $\mathrm{Ca}(\mathrm{OH})_{2}$ & single & MTA & PRP & 1.5 years \\
\hline Chen and Chen ${ }^{38}$ & $\begin{array}{l}\text { periapical healing and apex } \\
\text { formation in most cases }\end{array}$ & NA & $2.5 \% \mathrm{NaOCl}$ & $\mathrm{Ca}(\mathrm{OH})_{2}$ & multiple & MTA & blood clot & 1 year \\
\hline Estefan et al. ${ }^{39}$ & $\begin{array}{l}\text { periapical healing and an increase } \\
\text { in root apical narrowing }\end{array}$ & NA & $\begin{array}{l}2.5 \% \mathrm{NaOCl} \\
\text { and } \\
17 \% \text { EDTA }\end{array}$ & antibiotic & multiple & MTA & blood clot & 1 year \\
\hline Bukhari et al. ${ }^{40}$ & $\begin{array}{l}\text { absence of clinical signs and } \\
\text { symptoms; either a reduction } \\
\text { in size of periapical lesions, or } \\
\text { complete healing and apical } \\
\text { closure in most cases }\end{array}$ & $\begin{array}{l}\text { stage IV } \\
\quad(4) \\
\text { stage V } \\
(21)\end{array}$ & $\begin{array}{l}3 \% \mathrm{NaOCl} \\
\text { and } \\
17 \% \text { EDTA }\end{array}$ & antibiotic & multiple & $\begin{array}{l}\text { bioceramic } \\
\text { putty or } \\
\text { MTA }\end{array}$ & blood clot & $\begin{array}{c}2 \text { years } \\
(12) \\
4-6 \text { years } \\
(13)\end{array}$ \\
\hline Nosrat et al. ${ }^{41}$ & $\begin{array}{c}\text { root development evident in both } \\
\text { teeth }\end{array}$ & NA & $\begin{array}{l}1.25 \% \\
\mathrm{NaOCl} \text { and } \\
17 \% \text { EDTA }\end{array}$ & antibiotic & single & MTA & blood clot & 4 months \\
\hline Bezgin et al. ${ }^{42}$ & $\begin{array}{c}\text { apical closure in most cases and } \\
\text { evidence of bone healing in all } \\
\text { cases }\end{array}$ & $\begin{array}{l}\text { stage IV } \\
(6) \\
\text { stage V } \\
(13)\end{array}$ & $\begin{array}{l}2.5 \% \mathrm{NaOCl} \\
17 \% \mathrm{EDTA} \\
\text { and } \\
0.12 \% \mathrm{CHX}\end{array}$ & antibiotic & multiple & MTA & $\begin{array}{l}\text { blood clot } \\
\text { (9) } \\
\text { PRP } \\
(10)\end{array}$ & 1.5 years \\
\hline Narang et al. ${ }^{43}$ & $\begin{array}{l}\text { periapical healing and apical } \\
\text { closure in all cases }\end{array}$ & stage V & $2.5 \% \mathrm{NaOCl}$ & antibiotic & NR & $\begin{array}{l}\text { resin- } \\
\text { modified } \\
\text { GIC }\end{array}$ & $\begin{array}{l}\text { blood clot } \\
\text { (5) } \\
\text { PRP } \\
\text { (5) } \\
\text { PRF } \\
\text { (5) }\end{array}$ & 1.5 years \\
\hline McCabe $^{44}$ & $\begin{array}{l}\text { periapical healing and apical } \\
\text { closure }\end{array}$ & stage V & $\begin{array}{l}5 \% \mathrm{NaOCl} \\
\text { and } \\
17 \% \text { EDTA }\end{array}$ & NR & single & MTA & blood clot & 1.5 years \\
\hline
\end{tabular}

$\mathrm{NaOCl}$ - sodium hypochlorite; EDTA - ethylenediaminetetraacetic acid; $\mathrm{CHX}$ - chlorhexidine; $\mathrm{Ca}(\mathrm{OH})_{2}$ - calcium hydroxide; $\mathrm{MTA}$ - mineral trioxide aggregate; GIC - glass-ionomer cement; PRP - platelet-rich plasma; PRF - platelet-rich fibrin. 


\section{Secondary outcomes}

The reported secondary outcomes were variable and included an increase in root length and root wall thickness in 154 (62\%) cases. In addition, there was apical closure and complete root development in 112 (45\%) cases (Table 3).

\section{Etiology and diagnosis of successful RET cases}

In 97 successful cases, the gender of patients was reported (53 cases in males and 44 cases in females). Gender was not stated in the other 153 cases (Table 2). A total of 110 successful RET cases occurred in anterior teeth and 54 in posterior teeth. The tooth location was not indicated in the other 86 cases (Table 2). The etiology for the initiation of RET was not reported in 124 successful cases. On the other hand, a total of 126 successful RET cases reported the etiology resulting in the initiation of RET. Sixty-two (49\%) of these cases were caused by dental trauma, 30 (24\%) by dental caries, 29 (23\%) by dens evaginatus, 4 (3\%) by dens invaginatus, and $1(0.8 \%)$ by dental fracture (Table 2$)$. All successful cases reported a pulpal diagnosis with necrotic pulp, except for 1 case, ${ }^{36}$ which reported previously initiated therapy. Most of the successful cases that reported a periapical diagnosis had some type of apical pathosis. Only 2 cases reported normal apical tissue, 3 cases were diagnosed with an acute apical abscess, 11 cases reported a diagnosis of a chronic apical abscess, 13 cases were diagnosed with asymptomatic apical periodontitis, and 4 cases were diagnosed with symptomatic apical periodontitis. Also, 64 cases were reported "with apical pathosis" and 144 cases were reported "with or without apical pathosis" in the studies, without specifically identifying the type of apical pathology. Only 9 cases did not report an apical diagnosis (Table 2).

\section{Irrigation protocol used in successful RET cases}

The use of sodium hypochlorite $(\mathrm{NaOCl})$ as the main irrigation solution with a concentration ranging from $1 \%$ to $5.25 \%$ was reported in 249 (99.6\%) successful RET cases, while $1(0.4 \%)$ case $^{30}$ used $10 \mathrm{~mL}$ sterile saline and $20 \%$ ethylenediaminetetraacetic acid (EDTA). Nineteen (8\%) cases used chlorhexidine ( $\mathrm{CHX})$ irrigation with a concentration of $0.12 \%$. A total of 128 (51\%) successful cases reported that $\mathrm{NaOCl}$ was the only irrigation solution used in the procedure, 102 (41\%) of the successful cases used various combinations of $\mathrm{NaOCl}$ and EDTA, and 19 (8\%) of the successful cases used various combinations of $\mathrm{NaOCl}$, EDTA and CHX. A total of 128 (51\%) successful RET cases did not use EDTA in their irrigation protocols (Table 3 ). The type of intracanal medicament was reported in 249 (99.6\%) successful cases, and only 1 (0.4\%) case $^{43}$ did not report the type of intracanal medicament used in the procedure. Calcium hydroxide $\left(\mathrm{Ca}(\mathrm{OH})_{2}\right)$ was the only intracanal medicament used in 30 (12\%) cases, and 2 cases $(0.8 \%)$ used a combination of an antibiotic and $\mathrm{Ca}(\mathrm{OH})_{2}$. Twenty-six (10\%) cases used an antibiotic combination or $\mathrm{Ca}(\mathrm{OH})_{2}$ in their studies, and 191 (76\%) successful cases reported that an antibiotic was the only intracanal medicament used in the regenerative endodontic procedure (Table 3). In 15 (6\%) cases, RET was finished in a single visit, whereas in 220 (88\%) cases, the completion of RET required multiple visits. Only for 15 (6\%) successful cases, the number of visits was not reported (Table 3).

\section{Scaffold and capping materials used in successful RET cases}

A total of 214 (86\%) successful cases used a blood clot as a scaffold. Thirty-one (12\%) successful cases reported the use of platelet-rich plasma (PRP) in the RET protocol and $5(2 \%)$ successful cases reported the use of plateletrich fibrin (PRF) (Table 3). A total of 186 (74\%) successful cases reported that mineral trioxide aggregate (MTA) was the only capping material used in the procedure. Twentyfour (10\%) cases used glass-ionomer cement (GIC), 25 (10\%) cases used various combinations of capping materials with bioceramic putty or MTA and $15(6 \%)$ cases used resin-modified GIC in their RET (Table 3).

\section{Signs of success and root development stage of successful RET cases}

The reported time elapsed between the initiation of RET and the identification of successful cases ranged from 2 months to 8 years (Table 3 ). Three (1\%) successful cases were detected less than 1 year after the initiation of RET, 84 (34\%) successful cases were detected immediately after 1 year since the initiation of RET, 38 (15\%) successful cases were detected 1.5 years after the initiation of RET, 14 (6\%) successful cases were detected 2 years after the initiation of RET, and 98 (39\%) successful cases were detected at least 2.5 years after the initiation of RET. Only 13 (5\%) successful cases ranged the time of success from 1 year to 8 years, without specifically identifying the time for each case (Table 3). A total of 239 (96\%) successful cases reported the healing/absence of different periapical lesions as the main sign of success (Table 3). Due to the instructive radiographic features of Cvek's classification and the fact that it illustrates clinical outcomes better than other classification schemes, ${ }^{45}$ Cvek's classification was used in this review to determine the root maturation stage for all of the successful RET cases. The distribution of root development stages according to Cvek's classification was as follows: stage V (completed apical closure) was reported as a sign of success in 112 (45\%) cases; stage IV was reported in 34 (14\%) cases; stage III was reported in $8(3 \%)$ cases; and for $96(38 \%)$ cases, the root development stage was not reported (Table 3). 


\section{Assessment of the risk of bias of the included studies}

The Cochrane Collaboration's tool for determining the risk of bias was used for all the studies included in this review. ${ }^{27}$ The 2 authors integrated the information and summarized the risk of bias associated with the included studies. The majority of studies had a low risk of bias concerning the following domains: blinding of outcome assessment (94\%); incomplete outcome data (66\%); selective reporting (77\%); and other bias (88\%). All of the studies presented a low risk of bias (100\%) with regard to the random sequence generation, allocation concealment, and blinding of participants and personnel domains (Fig. 2). In summary, among the 18 studies assessed for the overall risk of bias, not one study was classified as having a high risk of bias; 11 (61\%) studies were considered to have a low risk of bias and 7 (39\%) studies had an unclear risk of bias (Fig. 3). The scoring of unclear risk of bias was given to 7 studies, as there was not enough information to make a clear judgment concerning the following domains: blinding of outcome assessment; incomplete outcome data; selective reporting; and other bias (Fig. 3). A review of the authors' judgments about each risk of bias domain is presented in Fig. 2. Finally, the risk of bias for each study is presented in Fig. 3.

\section{Discussion}

This systematic review was carried out to summarize and appraise all studies published within the last 6 years that were relevant to our study aim. It gathered all recent clinical studies that investigated the factors influencing RET outcomes for successful cases. This study demonstrates a comprehensive set of evidence extracted from 18 articles that fulfilled our inclusion and exclusion criteria. To date, 2 systematic reviews have discussed the signs of success in different human and animal studies concerning the preoperative factors and postoperative factors in
RET (Table 4). ${ }^{24,25}$ Alghamdi and Alqurashi concluded in their systematic review that most of the retrieved studies about RET suggested its effectiveness in periapical healing and the formation of apical closure in immature necrotic teeth. ${ }^{25}$ In addition, Torabinejad et al. demonstrated in their systematic review that the primary goal of RET could be reliably achieved with high probability (91-94\% of periapical healing). ${ }^{24}$ This clearly shows consistency in the conclusions drawn by the previously published systematic reviews. In agreement with those findings, 239 (96\%) cases in this review showed the healing/ absence of different periapical lesions after RET in human subjects. Also, the 2 abovementioned articles covered the period between 1966 and 2019 (Table 4), while our systematic review covered all eligible articles published within the last 6 years (Table 2 and Table 3 ).

In our systematic review, most of the successful cases mentioned the etiology for the initiation of RET (Table 2). Dental trauma accounted for $49 \%$ of these cases as the main etiology for the initiation of RET, followed by the existence of dental caries (24\%) and dens evaginatus (23\%). These outcomes are in agreement with a previously published review, which found that the etiology of $30 \%$ of all cases treated with RET was dental trauma, followed by the presence of dens evaginatus (22\%). ${ }^{46}$ However, for the majority of successful RET cases, the type of traumatic injury was not reported. ${ }^{46}$ One clinical study concluded that there were significantly better outcomes for RET cases with an etiology of dens evaginatus as compared to dental trauma. ${ }^{2}$ The current systematic review found that the healing/absence of periapical lesions was the main presentation of RET success in $96 \%$ of cases, as one of the signs of RET success (Table 3 ). In addition to other signs of success for these RET cases (Table 3), apical closure formation is the secondary goal of RET and the second sign of success in $45 \%$ of successful RET cases (Table 3 ) in comparison with other traditional treatment options for the management of immature necrotic teeth, including periapical surgery and apexification.

Random sequence generation

Allocation concealment

Blinding of participants and personnel

Blinding of outcome assessment Incomplete outcome data

Selective reporting

Other sources of bias

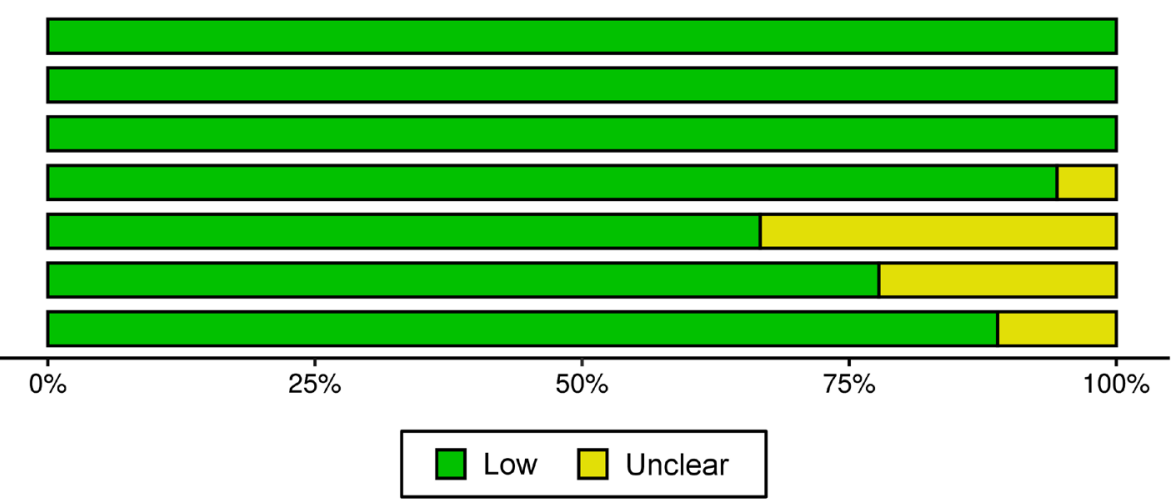

Fig. 2. Risk of bias graph

The review authors'judgments about each risk of bias item are presented as percentages across all the included studies. Green and yellow refer to a low risk of bias and an unclear risk of bias, respectively. 
Risk of bias

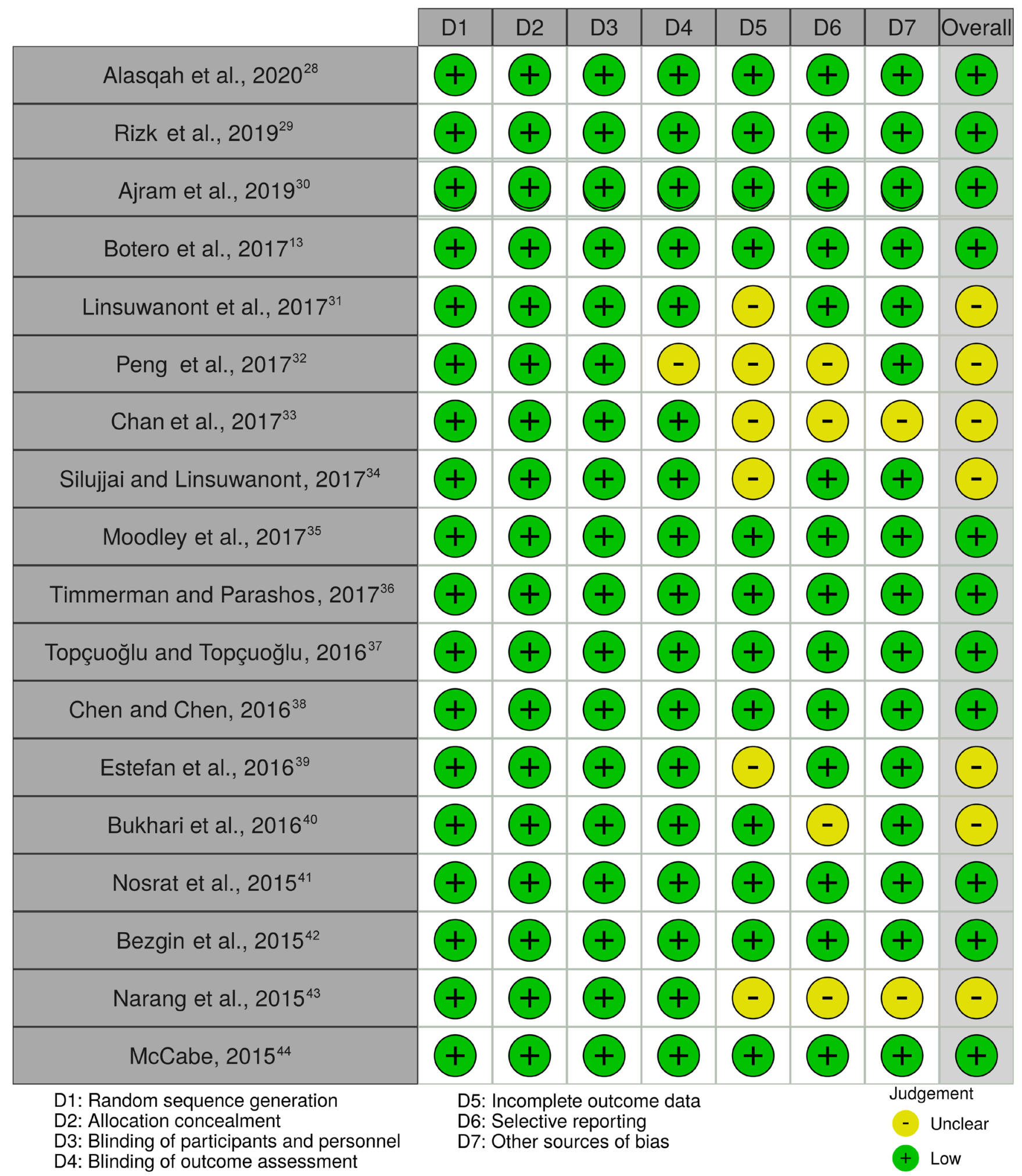

Fig. 3. Risk of bias summary

The review authors'judgments about each risk of bias item for each included study. Green and yellow refer to a low risk of bias and an unclear risk of bias, respectively.

Other publications point out that maintaining a high level of disinfection intra/postoperatively in RET is important to enhance RET outcomes. ${ }^{2,47-50}$ Antibiotic medicaments and high-concentration irrigation solutions are recommended to improve the irrigation protocols during $\mathrm{RET}$, since $\mathrm{NaOCl},{ }^{51} \mathrm{CHX},{ }^{52}$ and antibiotic intracanal medicaments ${ }^{53}$ have concentration-dependent antibacterial effects that contribute to reducing different 
Table 4. Summary of the 2 systematic reviews included in the present review

\begin{tabular}{|c|c|c|c|c|}
\hline Authors & Year & $\begin{array}{l}\text { Number } \\
\text { of studies } \\
\text { included }\end{array}$ & Method summary & Main conclusions \\
\hline Alghamdi and Alqurashi ${ }^{25}$ & 2020 & 46 studies & $\begin{array}{l}\text { The systematic review presents and summarizes } \\
\text { human and animal studies performed from } 2009 \\
\text { to } 2019 \text { retrieved by means of the electronic search } \\
\text { of } 2 \text { databases (PubMed and Google Scholar). }\end{array}$ & $\begin{array}{l}\text { RET was more efficient in treating immature } \\
\text { necrotic \$permanent teeth and offered a greater } \\
\text { advantage, which should lead to wider acceptance } \\
\text { among endodontists with regard to effective results } \\
\text { in comparison with other treatment options. }\end{array}$ \\
\hline Torabinejad et al. ${ }^{24}$ & 2017 & $\begin{array}{l}144 \\
\text { studies }\end{array}$ & $\begin{array}{l}\text { The systematic review presents and summarizes } \\
\text { in vivo human clinical studies performed from } \\
1966 \text { to November } 2016 \text { retrieved by means of the } \\
\text { electronic search of } 3 \text { databases (PubMed, Web } \\
\text { of Science and Cochrane Library). }\end{array}$ & $\begin{array}{c}\text { The treatment of immature teeth with pulp necrosis } \\
\text { with the use of MTA or RET shows high survival and } \\
\text { success rates. }\end{array}$ \\
\hline
\end{tabular}

biofilms produced by endodontic pathogens. However, the main irrigation predicament in RET is that lower concentrations of various intracanal medicaments and irrigation solutions are advised to preserve the survival of stem cells from apical papillae. ${ }^{54-57}$ Thus, different irrigation approaches should be examined in an endeavor to reach lower concentrations of antibacterial irrigations and medicaments that can still maintain a high level of irrigation.

In our systematic review, most of the successful RET cases had a follow-up period ranging from 2 months to 8 years. In contrast, there is a previous review that retrieved 18 successful cases with RET and reported the history of pulp necrosis no longer than half a year. ${ }^{58}$ Also, in the present study, the time elapsed between the initiation of RET and the recognition of successful RET was exactly 1 year in 34\% of successful RET cases. Furthermore, 39\% of all the included successful RET cases were identified at least 2.5 years after the initiation of RET. Some of the successful RET cases reported evidence of initial favorable outcomes, such as the resolution of radiographic lesions, ${ }^{34,35,41}$ apical closure, ${ }^{35,41}$ and an increase in root length and root thickness..$^{34,35,41}$ Several case reports and retrospective clinical studies in the literature have reported a high success rate for RET after a maximum follow-up period of 12-19 months. ${ }^{1,2,12,42,59}$ In one of the systematic reviews, they calculated the average follow-up time of RET studies to be 16.7 months. ${ }^{24}$

In our systematic review, 15 successful RET cases were completed in a single visit with different intracanal medicaments used. ${ }^{13,37,41,44}$ These results are in agreement with a previously published case report, in which RET was performed in a single visit and was reported to be successful. ${ }^{60}$ A total of $74 \%$ of successful RET cases reported the use of MTA as the main capping material in their studies. Other studies used different capping materials, including GIC (10\% of cases) and resin-modified GIC (6\% of cases). A comparative systematic review found that the pooled success rates for MTA apical plugs and RET were $94.6 \%$ and $91.3 \%$, respectively. ${ }^{24}$

In summary, this systematic review was developed based on the comparison of the impact of the preopera- tive and postoperative factors on eventual treatment success in RET (Table 2 and Table 3). Some studies used radiographic evidence of completed apical closure and the healing of periapical lesions to define the successful outcomes of RET cases. There is heterogeneity in the RET protocols used to manage the cases reported in this review. Thus, following the updated evidence-based clinical considerations from the American Association of Endodontists (AAE), it is compulsory to reduce variability in RET protocols. ${ }^{57}$ Finally, it can be concluded that RET can be a useful tool in the field of endodontics along with other approaches in treating children and young adult patients with immature necrotic teeth. The successful clinical RET cases discussed in this review can be considered as highlevel evidence in the field of endodontics with regard to patients with immature necrotic-pulp teeth. Based on the present evidence, it seems that the treatment of immature teeth with pulp necrosis by means of RET provides high survival and success rates.

\section{Study strengths and limitations}

This study summarized and appraised all peer-reviewed studies published within the last 6 years that fulfilled both our inclusion and exclusion criteria. To our knowledge, this is the only systematic review that covered the topic of the different factors influencing RET outcomes for successful cases in depth. The systematic reviews conducted by Alghamdi and Alqurashi ${ }^{25}$ and Torabinejad et al. ${ }^{24}$ covered the periods of 2009-2019 and 1966-2016, respectively (Table 4). Our review used PubMed and Google Scholar as search engines. One advantage of using Google Scholar is that it ensures that reviewers do not miss any relevant research published in journals that are still not cited in PubMed. Unfortunately, due to the heterogeneity of the influencing factors in the existing human studies, we were restricted to performing a systematic review without a meta-analysis. Finally, although studies conducted on human subjects were part of the inclusion criteria, we unfortunately could find but a few published successful case studies that used RET to treat children and young adult patients with immature necrotic teeth. 


\section{Conclusions}

Several RET cases of immature necrotic teeth were reported in this review. These cases provide clinical outcomes, such as apical closure formation, the healing of different forms of apical pathology as well as a significant follow-up duration. Thus, prudent case selection and excellent operative protocols are considered to be essential to achieve the successful outcomes of the RET procedure. Collectively, this systematic review showed that the healing/absence of periapical pathology was the main measure of success in $96 \%$ of successful RET cases. Furthermore, $45 \%$ of successful RET cases presented with root development maturation Stage V, and 39\% of successful RET cases were identified at least 2.5 years after the initiation of RET. Future studies should describe a variety of relevant data, including preoperative, intraoperative and postoperative factors, to provide a better understanding of successful cases after RET.

\section{ORCID iDs}

Faisal Alghamdi (1) https://orcid.org/0000-0003-2086-0772 Mohammed Alsulaimani (1) https://orcid.org/0000-0002-3058-9499

\section{References}

1. Nagata JY, Figueiredo de Almeida Gomes BP, Rocha Lima TF, et al. Traumatized immature teeth treated with 2 protocols of pulp revascularization. J Endod. 2014;40(5):606-612. doi:10.1016/j.joen.2014.01.032

2. Lin J, Zeng $Q$, Wei $X$, et al. Regenerative endodontics versus apexification in immature permanent teeth with apical periodontitis: A prospective randomized controlled study. J Endod. 2017;43(11):1821-1827. doi:10.1016/j.joen.2017.06.023

3. Geisler TM. Clinical considerations for regenerative endodontic procedures. Dent Clin North Am. 2012;56(3):603-626. doi:10.1016/j.cden.2012.05.010

4. Hargreaves KM, Diogenes A, Teixeira FB. Treatment options: Biological basis of regenerative endodontic procedures. J Endod. 2013;39(3 Suppl):S30-S43. doi:10.1016/j.joen.2012.11.025

5. Banchs F, Trope M. Revascularization of immature permanent teeth with apical periodontitis: New treatment protocol? J Endod. 2004;30(4):196-200. doi:10.1097/00004770-200404000-00003

6. Ostby BN. The role of the blood clot in endodontic therapy. An experimental histologic study. Acta Odontol Scand. 1961;19:324-353. PMID:14482575.

7. Hargreaves KM, Cohen S, eds.; Berman LH, web ed. Cohen's Pathways of the Pulp. 10 $0^{\text {th }}$ ed. St. Louis, MO: Mosby Elsevier; 2011.

8. Thibodeau B, Trope M. Pulp revascularization of a necrotic infected immature permanent tooth: Case report and review of the literature. Pediatr Dent. 2007;29(1):47-50. PMID:18041512.

9. Wigler R, Kaufman AY, Lin S, Steinbock N, Hazan-Molina H, Torneck CD. Revascularization: A treatment for permanent teeth with necrotic pulp and incomplete root development. J Endod. 2013;39(3):319-326. doi:10.1016/j.joen.2012.11.014

10. Huang GTJ. A paradigm shift in endodontic management of immature teeth: Conservation of stem cells for regeneration. J Dent. 2008;36(6):379-386. doi:10.1016/j.jdent.2008.03.002

11. Yamauchi $N$, Yamauchi $S$, Nagaoka $H$, et al. Tissue engineering strategies for immature teeth with apical periodontitis. J Endod. 2011;37(3):390-397. doi:10.1016/j.joen.2010.11.010

12. Nagy MM, Tawfik HE, Rahman Hashem AA, Abu-Seida AM. Regenerative potential of immature permanent teeth with necrotic pulps after different regenerative protocols. J Endod. 2014;40(2):192-198. doi:10.1016/j.joen.2013.10.027
13. Botero TM, Tang X, Gardner R, Hu JCC, Boynton JR, Holland GR. Clinical evidence for regenerative endodontic procedures: Immediate versus delayed induction? J Endod. 2017;43(9S):S75-S81. doi:10.1016/j.joen.2017.07.009

14. Kim SG, Malek M, Sigurdsson A, Lin LM, Kahler B. Regenerative endodontics: A comprehensive review. Int Endod J. 2018;51(12):1367-1388. doi:10.1111/iej.12954

15. Iwaya SI, Ikawa M, Kubota M. Revascularization of an immature permanent tooth with apical periodontitis and sinus tract. Dent Traumatol. 2001;17(4):185-187. doi:10.1034/j.1600-9657.2001.017004185.x

16. Bose R, Nummikoski $P$, Hargreaves K. A retrospective evaluation of radiographic outcomes in immature teeth with necrotic root canal systems treated with regenerative endodontic procedures. J Endod. 2009;35(10):1343-1349. doi:10.1016/j.joen.2009.06.021

17. Saoud TMA, Zaazou A, Nabil A, Moussa S, Lin LM, Gibbs JL. Clinical and radiographic outcomes of traumatized immature permanent necrotic teeth after revascularization/revitalization therapy. J Endod. 2014;40(12):1946-1952. doi:10.1016/j.joen.2014.08.023

18. Cehreli ZC, Isbitiren B, Sara S, Erbas G. Regenerative endodontic treatment (revascularization) of immature necrotic molars medicated with calcium hydroxide: A case series. J Endod. 2011;37(9):1327-1330. doi:10.1016/j.joen.2011.05.033

19. Chen MYH, Chen KL, Chen CA, Tayebaty F, Rosenberg PA, Lin LM. Responses of immature permanent teeth with infected necrotic pulp tissue and apical periodontitis/abscess to revascularization procedures. Int Endod J. 2012;45(3):294-305. doi:10.1111/j.1365-2591.2011.01978.x

20. Chueh LH, Ho YC, Kuo TC, Lai WH, Chen YHM, Chiang CP. Regenerative endodontic treatment for necrotic immature permanent teeth. J Endod. 2009;35(2):160-164. doi:10.1016/j.joen.2008.10.019

21. Jeeruphan T, Jantarat J, Yanpiset K, Suwannapan L, Khewsawai $P$, Hargreaves KM. Mahidol study 1: Comparison of radiographic and survival outcomes of immature teeth treated with either regenerative endodontic or apexification methods: A retrospective study. J Endod. 2012;38(10):1330-1336. doi:10.1016/j.joen.2012.06.028

22. Jung IY, Lee SJ, Hargreaves KM. Biologically based treatment of immature permanent teeth with pulpal necrosis: A case series. J Endod. 2008;34(7):876-887. doi:10.1016/j.joen.2008.03.023

23. Lee BN, Moon JW, Chang HS, Hwang IN, Oh WM, Hwang YC. A review of the regenerative endodontic treatment procedure. Restor Dent Endod. 2015;40(3):179-187. doi:10.5395/rde.2015.40.3.179

24. Torabinejad M, Nosrat A, Verma P, Udochukwu O. Regenerative endodontic treatment or mineral trioxide aggregate apical plug in teeth with necrotic pulps and open apices: A systematic review and meta-analysis. J Endod. 2017;43(11):1806-1820. doi:10.1016/j.joen.2017.06.029

25. Alghamdi FT, Alqurashi AE. Regenerative endodontic therapy in the management of immature necrotic permanent dentition: A systematic review. ScientificWorldJournal. 2020;2020:7954357. doi:10.1155/2020/7954357

26. Moher D, Liberati A, Tetzlaff J, Altman DG; PRISMA group. Preferred reporting items for systematic reviews and metaanalyses: The PRISMA statement. PLoS Med. 2009;6(7):e1000097. doi:10.1371/journal.pmed.1000097

27. Higgins JPT, Deeks JJ, Altman DG; Cochrane Statistical Methods Group. Chapter 16: Special topics in statistics. In: Higgins JPT, Green S, eds. Cochrane Handbook for Systematic Reviews of Interventions. Version 5.1.0 (updated March 2011). The Cochrane Collaboration; 2011. www.handbook.cochrane.org. Accessed September 1, 2020.

28. Alasqah M, Raja Khan SI, Alfouzan K, Jamleh A. Regenerative endodontic management of an immature molar using calcium hydroxide and triple antibiotic paste: A two-year follow-up. Case Rep Dent. 2020;2020:9025847. doi:10.1155/2020/9025847

29. Rizk HM, Salah Al-Deen MS, Emam AA. Regenerative endodontic treatment of bilateral necrotic immature permanent maxillary central incisors with platelet-rich plasma versus blood clot: A split mouth double-blinded randomized controlled trial. Int J Clin Pediatr Dent. 2019;12(4):332-339. doi:10.5005/jp-journals-10005-1656

30. Ajram J, Khalil I, Gergi R, Zogheib C. Management of an immature necrotic permanent molar with apical periodontitis treated by regenerative endodontic protocol using calcium hydroxide and MM-MTA: A case report with two years follow up. Dent J (Basel). 2019;7(1):1. doi:10.3390/dj7010001 
31. Linsuwanont $P$, Sinpitaksakul P, Lertsakchai T. Evaluation of root maturation after revitalization in immature permanent teeth with nonvital pulps by cone beam computed tomography and conventional radiographs. Int Endod J. 2017;50(9):836-846. doi:10.1111/iej.12705

32. Peng $C$, Yang $Y$, Zhao $Y$, et al. Long-term treatment outcomes in immature permanent teeth by revascularisation using MTA and GIC as canal-sealing materials: A retrospective study. Int J Paediatr Dent. 2017;27(6):454-462. doi:10.1111/ipd.12282

33. Chan EKM, Desmeules M, Cielecki M, Dabbagh B, Ferraz Dos Santos B. Longitudinal cohort study of regenerative endodontic treatment for immature necrotic permanent teeth. J Endod. 2017;43(3):395-400. doi:10.1016/j.joen.2016.10.035

34. Silujjai J, Linsuwanont $P$. Treatment outcomes of apexification or revascularization in nonvital immature permanent teeth: $A$ retrospective study. J Endod. 2017;43(2):238-245. doi:10.1016/j.joen.2016.10.030.

35. Moodley DS, Peck C, Moodley T, Patel N. Management of necrotic pulp of immature permanent incisor tooth: A regenerative endodontic treatment protocol: Case report. S Afr Dent J. 2017;72(3):122-125.

36. Timmerman A, Parashos P. Delayed root development by displaced mineral trioxide aggregate after regenerative endodontics: A case report. J Endod. 2017;43(2):252-256. doi:10.1016/j.joen.2016.10.024

37. Topçuoğlu G, Topçuoğlu HS. Regenerative endodontic therapy in a single visit using platelet-rich plasma and biodentine in necrotic and asymptomatic immature molar teeth: A report of 3 cases. J Endod. 2016;42(9):1344-1346. doi:10.1016/j.joen.2016.06.005

38. Chen SJ, Chen LP. Radiographic outcome of necrotic immature teeth treated with two endodontic techniques: A retrospective analysis. Biomed J. 2016;39(5):366-371. doi:10.1016/j.bj.2015.12.006

39. Estefan BS, El Batouty KM, Nagy MM, Diogenes A. Influence of age and apical diameter on the success of endodontic regeneration procedures. J Endod. 2016;42(11):1620-1625. doi:10.1016/j.joen.2016.06.020

40. Bukhari S, Kohli MR, Setzer F, Karabucak B. Outcome of revascularization procedure: A retrospective case series. J Endod. 2016;42(12):1752-1759. doi:10.1016/j.joen.2016.06.021

41. Nosrat A, Kolahdouzan A, Hosseini F, Mehrizi EA, Verma P, Torabinejad $M$. Histologic outcomes of uninfected human immature teeth treated with regenerative endodontics: 2 case reports. J Endod. 2015;41(10):1725-1729. doi:10.1016/j.joen.2015.05.004

42. Bezgin T, Yilmaz AD, Celik BN, Kolsuz ME, Sonmez H. Efficacy of platelet-rich plasma as a scaffold in regenerative endodontic treatment. J Endod. 2015;41(1):36-44. doi:10.1016/j.joen.2014.10.004

43. Narang I, Mittal N, Mishra N. A comparative evaluation of the blood clot, platelet-rich plasma, and platelet-rich fibrin in regeneration of necrotic immature permanent teeth: A clinical study. Contemp Clin Dent. 2015;6(1):63-68. doi:10.4103/0976-237X.149294

44. McCabe P. Revascularization of an immature tooth with apical periodontitis using a single visit protocol: A case report. Int Endod J. 2015;48(5):484-497. doi:10.1111/iej.12344

45. Cvek M. Prognosis of luxated non-vital maxillary incisors treated with calcium hydroxide and filled with gutta-percha. A retrospective clinical study. Endod Dent Traumatol. 1992;8(2):45-55. doi:10.1111/j.1600-9657.1992.tb00228.x

46. Diogenes A, Henry MA, Teixeira FB, Hargreaves KM. An update on clinical regenerative endodontics. Endod Topics. 2013;28(1):2-23. doi:10.1111/etp.12040

47. Lin LM, Shimizu E, Gibbs JL, Loghin S, Ricucci D. Histologic and histobacteriologic observations of failed revascularization/revitalization therapy: A case report. J Endod. 2014;40(2):291-295. doi:10.1016/j.joen.2013.08.024

48. Chaniotis A. Treatment options for failing regenerative endodontic procedures: Report of 3 cases. J Endod. 2017;43(9):1472-1478. doi:10.1016/j.joen.2017.04.015

49. Žižka R, Buchta T, Voborná I, Harvan L, Šedý J. Root maturation in teeth treated by unsuccessful revitalization: 2 case reports. J Endod. 2016;42(5):724-729. doi:10.1016/j.joen.2016.02.004

50. Verma P, Nosrat A, Kim JR, et al. Effect of residual bacteria on the outcome of pulp regeneration in vivo. J Dent Res. 2017;96(1):100-106. doi:10.1177/0022034516671499

51. del Carpio-Perochena A, Bramante CM, de Andrade FB, et al. Antibacterial and dissolution ability of sodium hypochlorite in different pHs on multi-species biofilms. Clin Oral Investig. 2015;19(8):2067-2073. doi:10.1007/s00784-015-1431-6
52. Ma J, Tong $\mathrm{Z}$, Ling J, Liu $\mathrm{H}$, Wei $X$. The effects of sodium hypochlorite and chlorhexidine irrigants on the antibacterial activities of alkaline media against Enterococcus faecalis. Arch Oral Biol. 2015;60(7):1075-1081. doi:10.1016/j.archoralbio.2015.04.008

53. Tagelsir A, Yassen GH, Gomez GF, Gregory RL. Effect of antimicrobials used in regenerative endodontic procedures on 3-weekold Enterococcus faecalis biofilm. J Endod. 2016;42(2):258-262. doi:10.1016/j.joen.2015.09.023

54. Trevino EG, Patwardhan AN, Henry MA, et al. Effect of irrigants on the survival of human stem cells of the apical papilla in a plateletrich plasma scaffold in human root tips. J Endod. 2011;37(8):1109-1115. doi:10.1016/j.joen.2011.05.013

55. Martin DE, De Almeida JFA, Henry MA, et al. Concentrationdependent effect of sodium hypochlorite on stem cells of apical papilla survival and differentiation. J Endod. 2014;40(1):51-55. doi:10.1016/j.joen.2013.07.026

56. Althumairy RI, Teixeira FB, Diogenes A. Effect of dentin conditioning with intracanal medicaments on survival of stem cells of apical papilla. J Endod. 2014;40(4):521-525. doi:10.1016/j.joen.2013.11.008

57. American Association of Endodontists. AAE clinical considerations for a regenerative procedure. Revised 4/1/2018. https://www.aae. org/specialty/wp-content/uploads/sites/2/2018/06/ConsiderationsForRegEndo_AsOfApril2018.pdf. Accessed September 23, 2018.

58. Nosrat A, Homayounfar N, Oloomi K. Drawbacks and unfavorable outcomes of regenerative endodontic treatments of necrotic immature teeth: A literature review and report of a case. J Endod. 2012;38(10):1428-1434. doi:10.1016/j.joen.2012.06.025

59. Kahler B, Mistry S, Moule A, et al. Revascularization outcomes: A prospective analysis of 16 consecutive cases. J Endod. 2014;40(3):333-338. doi:10.1016/j.joen.2013.10.032

60. Shin SY, Albert JS, Mortman RE. One step pulp revascularization treatment of an immature permanent tooth with chronic apical abscess: A case report. Int Endod J. 2009;42(12):1118-1126. doi:10.1111/j.1365-2591.2009.01633.x 